\title{
Full-Thickness Macular Hole Progressing from Lamellar Macular Hole with Epiretinal Proliferation
}

\author{
Salim Zafar Asaad \\ Burjeel Speciality Hospital, Sharjah, United Arab Emirates
}

\section{Keywords}

Epiretinal proliferation - Lamellar macular hole · Full-thickness macular hole

\begin{abstract}
Foveoschisis in association with contractile epiretinal membrane is frequently misdiagnosed as lamellar macular hole (LMH). The progression of true $\mathrm{LMH}$ with associated epiretinal proliferation to full-thickness macular hole (FTMH) is probably underestimated. We report a case of bilateral FTMH progressing from $\mathrm{LMH}$ with epiretinal proliferation. A 72-year-old male presented with increased blurring of vision in left eye. Visual acuity was 20/40 in right and 20/50 in left eye. Examination showed FTMH with epiretinal proliferation in left eye and LMH with epiretinal proliferation in right eye. Surgery of the left eye achieved successful closure of macular hole with improvement in vision. He presented again a month later with further deterioration of vision in right eye. Examination revealed FTMH in right eye with epiretinal proliferation. Surgery of the right eye also achieved optimum functional and morphological results. This case demonstrates that $\mathrm{LMH}$ with epiretinal proliferation may have a propensity to progress to FTMH.
\end{abstract}

(C) 2021 The Author(s).

Published by S. Karger AG, Basel

\section{Introduction}

There is currently no consensus about what constitutes "lamellar macular hole" (LMH) and its definition. The term alludes to a wide spectrum of macular pathomorphologies. Gass [1] first used the term to describe complication of cystoid macular edema after cataract extraction. In 2006, Witkin et al. [2] proposed optical coherence tomography (OCT) criteria for diagnosis of LMHs that were adopted by the International Vitreomacular Traction Study Group [3], which included irregular foveal contour, defect in the 
inner fovea, intraretinal split, and intact photoreceptors. But this definition does not address the associated preretinal tissue or status of ellipsoidal layer which influences clinical course and prognosis. Govetto et al. [4] described 2 subtypes of LMHs which are clinically distinct; the first "tractional" characterized by schitic separation of the neurosensory retina and the second "degenerative" characterized by intraretinal cavitation with ellipsoidal zone defect. They observed that tractional LMHs are associated with tractional epiretinal membrane (ERM) and degenerative LMHs with nontractional epiretinal proliferation. Many other authors have also described 2 types of preretinal tissue associated with LMHs. The first type referred to as tractional ERM [5]/normal ERM [6]/conventional ERM [7]/typical tractional ERM [8]/standard ERM [9] appears tomographically as an irregular hyper-reflective layer attached intermittently to the underlying retina and associated with tractional signs such as retinal wrinkling, thickening, and intraretinal cysts. The second type referred to as thick ERM (Witkins et al. [2])/dense ERM (Parolini et al. [5])/thicker ERM (Bottoni et al. [6])/lamellar hole-associated epiretinal proliferation or LHEP (Pang et al. [7])/atypical epiretinal tissue (Schumann et al. [8]) appears tomographically as a thick homogenous material of medium reflectivity universally adherent to the underlying retina and exhibits no evidence of traction.

Recently, Hubschman, Govetto, and other members of an international panel of vitreoretinal experts proposed that only lesions with apparent tissue loss should be considered as LMHs and, therefore, presence of foveal cavity with undermined edges mandatory for its diagnosis along with irregular foveal contour and other signs of foveal tissue loss like thinning and pseudooperculum [10]. Optional criteria suggested were epiretinal proliferation, ellipsoid line disruption, and foveal bump. This definition is similar to Govetto et al. [4] description in 2016 of degenerative LMH, one of their proposed subtypes of LMH. Their other proposed subtype tractional LMH does not involve any actual tissue loss, the pathomorphologic changes caused by traction, and therefore, the panel proposed to name it as "ERM foveoschisis." Hubschman et al. [10] also proposed that thick ERM/dense ERM/ atypical epiretinal tissue/LHEP be named "epiretinal proliferation." Although the term LHEP is widely used, we agree that it is not precise as the material has been found in conditions other than LMHs in FTMHs and at extrafoveal sites following trauma iatrogenic and accidental [7]. For this article, we have used the nomenclature proposed by Hubschman et al. [10] but understand that concepts, definitions, and terminology may evolve and change in future.

\section{Case Report}

A septuagenarian male presented with increased blurring of vision in left eye for 1 week. He said he had blurred vision in both eyes since about a year. Best-corrected visual acuity was 20/40 in right eye and 20/50 in left eye. Anterior examination showed pseudophakia in both eyes, was otherwise unremarkable. Fundus examination showed FTMH in left eye, LMH in right eye with epimacular material in both eyes. OCT of left eye showed irregular margins of the macular hole with intraretinal edema. Epiretinal proliferation recognized as a homogeneous, isoreflective layer covered by a thin hyper-reflective line was seen at the edges of the hole contiguous with inner retina. Early ERM without tractional signs was also seen more centrifugally (Fig. 1a). OCT of right eye showed disruption of ellipsoidal layer and external limiting membrane and a cavitated appearance of the retina temporally. Epiretinal proliferation was observed as a thick, homogeneous, isoreflective layer covered by a thin hyperreflective line was seen at the surface of the hole edges and seemed contiguous with inner

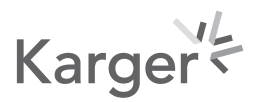



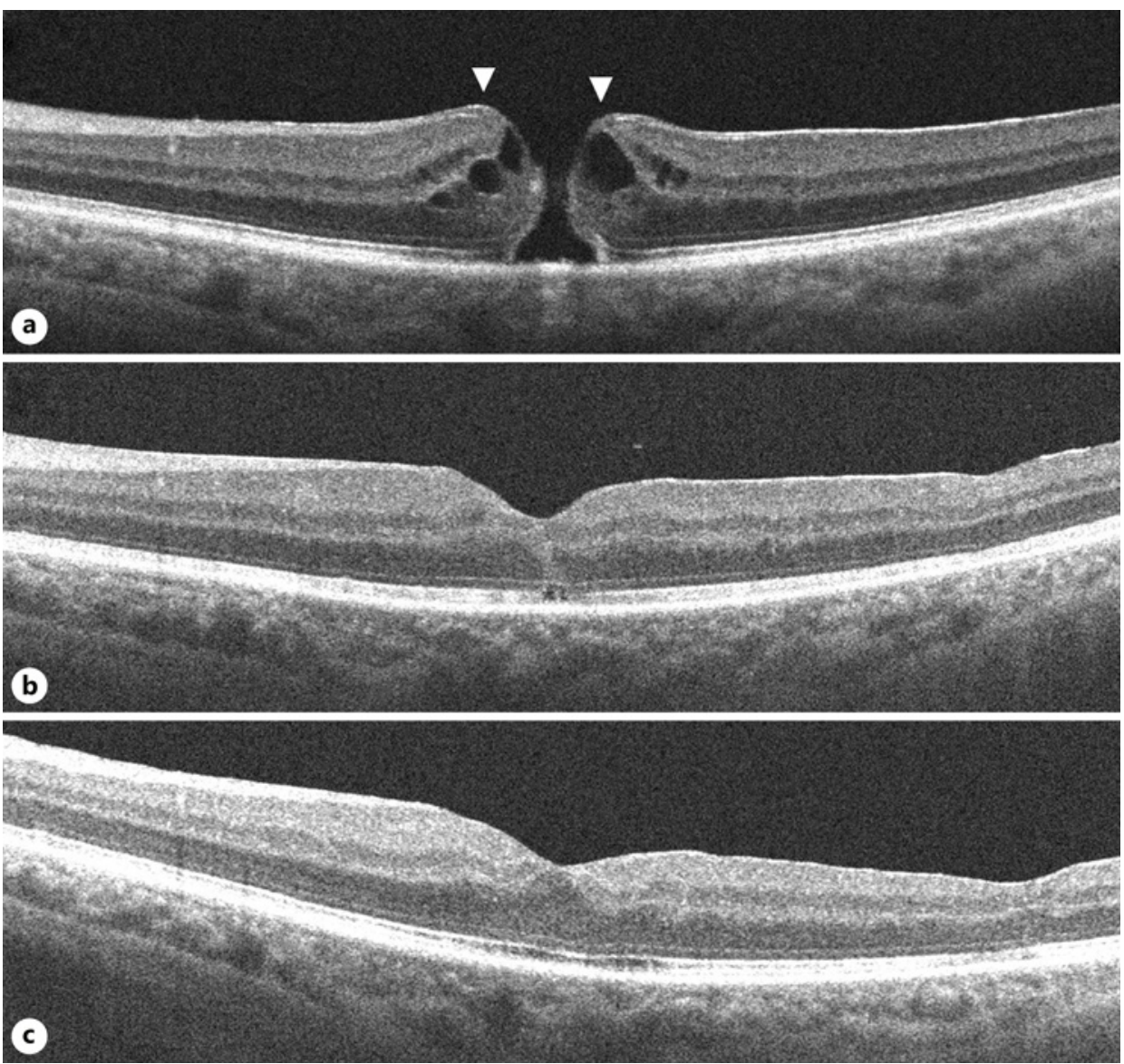

Fig. 1. OCT images of the left eye. a On presentation, a FTMH with epiretinal proliferation at its edges seen as a homogeneous, isoreflective layer covered by a thin hyper-reflective line contiguous with inner retina (arrows). b At one-month post-surgery, the macular hole is closed; a small ORD is seen. c At 2-month post-surgery, the ORD has resolved. FTMH, full-thickness macular hole; OCT, optical coherence tomography; ORD, outer retinal defect.

retina. ERM was seen centrifugally without tractional signs. The detached posterior hyaloid face was also visible in the scans (Fig. 2a, b).

The patient underwent pars plana vitrectomy with epiretinal proliferation and ERM peeling with internal limiting membrane (ILM) peeling with sulfur hexafluoride (SF6) in left eye. Successful closure of the macular hole was achieved. There was a small outer retinal defect (ORD) in the initial postoperative period which resolved spontaneously on follow-up (Fig. 1b, c). Visual acuity improved to 20/25 recorded 2-month postoperative. OCT of the right eye at this point showed further disorganization of central retina but visual acuity was maintained.

He next presented again a month later with further deterioration vision in right eye since 1 day. Best-corrected visual acuity was 20/60 in right eye and 20/25 in left eye. Examination showed a FTMH in right eye. OCT showed irregular edematous margins of the macular hole. Epiretinal proliferation seen at the hole edges appeared contiguous with the inner retina. ERM was also seen a bit centrifugally (Fig. 2c). He underwent pars plana vitrectomy with epiretinal proliferation and ERM peeling with ILM peeling with SF6 in the right eye which achieved successful closure of the hole. An ORD and subretinal fluid subfoveally was seen in/ during the initial postoperative period, but vision had improved to 20/32 (Fig. 2d). He was unable to follow-up after one month. 


\section{Case Reports in Ophthalmology}
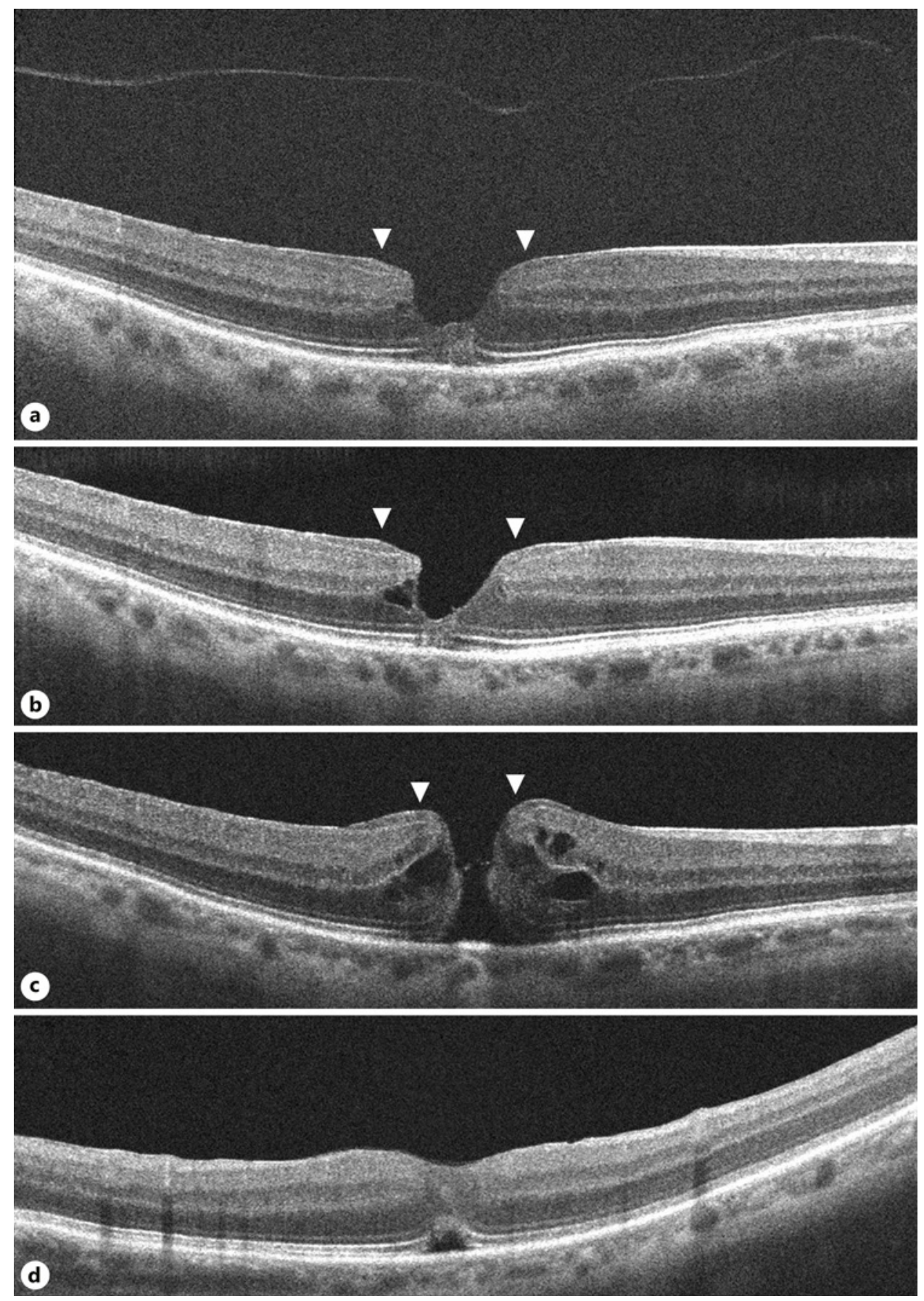

Fig. 2. OCT images of the right eye. a, b On presentation, shows a typical LMH with irregular foveal contour with thinning, foveal cavity, epiretinal proliferation, and disruption of ellipsoidal layer and external limiting membrane. Epiretinal proliferation is seen as a thick homogeneous, isoreflective layer covered by a thin hyper-reflective line at the edges of the hole contiguous with inner retina (arrows). The detached posterior hyaloid is also seen. c On occurrence of FTMH, epiretinal proliferation at the hole edges (arrows) appears contiguous with the inner retina (arrows). $\mathbf{d}$ At one-month post-surgery, the macular hole is closed; an ORD is seen. LMH, lamellar macular hole; FTMH, full-thickness macular hole; OCT, optical coherence tomography; ORD, outer retinal defect. 
Asaad: Full-Thickness Macular Hole and Epiretinal Proliferation

\section{Discussion}

LMHs are invariably accompanied by ERM and/or epiretinal proliferation and can occasionally progress to FTMH $[6,7,9,11-15]$. The pathogenesis of LMH progression to FTMH is poorly understood, but it is evident that the vitreous plays no role. Conversely, an idiopathic FTMH forms due to vitreofoveal traction with perifoveal vitreous detachment [16]. Thereafter, ERM and/or epiretinal proliferation may develop around and have an adjunctive role in enlarging it $[7,17]$. Thus, when a FTMH is observed along with ERM/epiretinal proliferation and PVD, it may not be obvious at first whether it occurred before the development of ERM/epiretinal proliferation or formed as a consequence of ERM/epiretinal proliferation with LMH. Tomographic images before the formation of FTMH if available would of course easily resolve this dilemma. Which in our case were available for the right eye documenting typical LMH before the occurrence of FTMH. Additionally, the scans also show detached posterior hyaloid ruling out its role in subsequent FTMH formation. There are other signs which may indicate that a FTMH has evolved from LMH. The configuration of these holes might be suggestive of pre-existing inner retinal defect, distinct from that of idiopathic FTMH [13]. Finally, the presence of epiretinal proliferation at the hole margins suggests it might have evolved from LMH as discussed earlier. In our case, OCT scans demonstrate the presence of epiretinal proliferation in both eyes. The clinical presentation, sequential images, and tomographic findings indicate that FTMH in both the eyes progressed from LMH with epiretinal proliferation.

ERM is seen more frequently than epiretinal proliferation and associated with a variety of clinical conditions. ERM appears as a whitish-gray translucent sheet on the retinal surface whereas epiretinal proliferation is typically not visible on ophthalmoscopy. On tomography, ERM appears as highly reflective usually thin line whereas epiretinal proliferation is seen as usually thick homogenous material of medium reflectivity. On cursory examination, the isoreflective epiretinal proliferation can be mistaken as part of the retina because of a thin hyperreflective line often covering it which is mistaken as ERM $[2,5,10]$. ERM is intermittently attached with frequent skip areas of contact with the underlying ILM and associated with other tractional signs such as retinal wrinkling, thickening, and intraretinal cysts. In contrast, epiretinal proliferation is universally adherent to the underlying retina and exhibits no evidence of traction. Epiretinal proliferation is only found in conditions with defects extending to the middle retinal layers and appears contiguous with them [7]. They have been seen associated with LMH, FTMH, and at sites of trauma iatrogenic or accidental. In LMHs, epiretinal proliferation is seen around/surrounding the edges of the defect and moulds with the inner retinal anatomy [7]. Both ERM and epiretinal proliferation can coexist manifesting mixed features $[4,7,9,10,12]$. In our case, tomography showed epiretinal proliferation at hole edges and ERM centrifugally in both eyes.

ERM traction can cause foveoschisis and the appearance of an irregular foveal contour that can be confused with LMH [10]. The pathogenesis is similar to that of macular pseudoholes where the morphologic changes are caused by tractional forces exerted by the overlying ERM [4]. ERM foveoschisis is associated with other tractional signs like retinal thickening, wrinkling, and intraretinal cysts. Foveoschisis in association with contractile ERM is frequently misdiagnosed as LMH.

Many studies have concluded that LMHs usually remain stable over time, very few evolving into FTMH $[2,6,7,9]$. We reviewed previously published literature on LMHs addressing natural history to compare their study cases to the current definition as proposed by Hubschman et al. [10]. Earlier studies mostly did not describe the morphology of LMH in their study cases, but later reports have usually specified the subtype of LMH or associated preretinal tissue making it possible to estimate the clinical course of true LMHs.

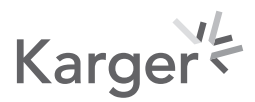


Witkin et al. [2] observed 19 eyes with LMH of which 6 had normal ERM and 11 had thickened ERM (likely similar to true LMH as defined by Hubschman et al. [10]). One eye progressed to a FTMH, but it is not specified what type of ERM it had. Bottoni et al. [6] followed 34 eyes with LMH with 2 different types of ERM, thicker (likely similar to true LMH), and normal (likely similar to ERM foveoschisis as defined by Hubschman et al. [10]). Of the 10 thicker ERMs and 24 normal ERMs, one of each type progressed to FTMH after 6and 15-month follow-up, respectively. Pang et al. [7] reviewed 2030 eyes of which LHEP (epiretinal proliferation) was found in 60 of 197 eyes with LMH and 8 of 99 eyes with FTMH. We think it is possible that the eyes with FTMH with epiretinal proliferation could have progressed from LMH with epiretinal proliferation. Recently formed idiopathic FTMH would not be expected to have any pre-macular tissue, though ERM or epiretinal proliferation may develop after some period of time. Our patient presented within a few days of experiencing deterioration of vision in left eye, presumably upon occurrence of FTMH. In another study, Pang et al. [11] compared 62 eyes with LMH with LHEP (epiretinal proliferation) and 83 eyes with LMH with conventional ERM (likely similar to ERM foveoschisis). During the mean follow-up period of 26 months, only one eye progressed to FTMH but it is not specified from which group; otherwise functionally and morphologically the LHEP group fared worse. Compera et al. [12] reported a case of LMH with LHEP (Epiretinal proliferation) that progressed to FTMH. Dell'Omo et al. [9] analyzed 84 eyes with LMH, of which 43 had standard ERM alone (likely similar to ERM foveoschisis), 11 had LHEP alone (likely similar to true LMH) and 30 had both. In the follow-up period, 3 eyes in the LHEP group progressed to FTMH.

Surgery for FTMH with epiretinal proliferation has not been reported much in the literature. Pang et al. [7] reported that in all the 3 cases of FTMH with LHEP (epiretinal proliferation) in their study group, there was successful macular hole closure after vitrectomy, ILM peel, intraocular tamponade, and postoperative posturing. They observed that it is more difficult to start peeling LHEP than typical ERM describing it is more elastic and typically yellow [7]. Peeling of epiretinal proliferation might be not as important for relieving retinal traction as peeling ERM but would have the function of allowing surgical access to the ILM [9]. Parolini et al. [5] observed dense ERM (epiretinal proliferation) intraoperatively as having a yellow dense appearance and a fluffy consistency which could be completely separated from the retina and the ILM. In our experience, surgery for FTMH is not much different than that for FTMH with ERM. Except for the part about peeling the epiretinal proliferation which felt more friable and pliant, neither does it stain as well with trypan blue. In our case, closure of FTMH with improvement in vision was achieved in both eyes after surgery despite ORDs in the initial postoperative period. The presence of central ORD with subretinal fluid on OCT after successful macular hole surgery is not uncommon but does not impede visual improvement and usually resolves in few weeks $[18,19]$.

This case and the analysis of previous reports by excluding tractional entities from their study population suggest that true LMHs have a worse functional, morphological outcome and may have a propensity to progress to FTMH. Prospective studies on the natural history of LMHs and evaluating the role of epiretinal proliferation in the progression to FTMH are warranted to improve the management of these lesions.

\section{Statement of Ethics}

This study complied with the tenets of the Declaration of Helsinki. Approval was obtained from Burjeel Hospital Institutional Review Board.

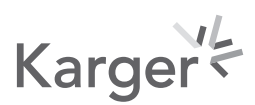




\section{Conflict of Interest Statement}

The author declares that he has no conflicting interests.

\section{Funding Sources}

None to declare.

\section{Author Contributions}

All the authors attest that they meet the current ICMJE criteria for authorship.

\section{Patient Consent}

Written informed consent was obtained from the patient to publish this case report and accompanying images. It is available for review by the journal editor.

\section{References}

1 Gass JD. Lamellar macular hole: a complication of cystoid macular edema after cataract extraction: a clinicopathologic case report. Trans Am Ophthalmol Soc. 1976;73:231-50.

2 Witkin AJ, Ko TH, Fujimoto JG, Schuman JS, Baumal CR, Rogers AH, et al. Redefining lamellar holes and the vitreomacular interface: an ultrahigh-resolution optical coherence tomography study. Ophthalmology. 2006 Mar;113(3):388-97.

3 Duker JS, Kaiser PK, Binder S, de Smet MD, Gaudric A, Reichel E, et al. The International Vitreomacular Traction Study Group classification of vitreomacular adhesion, traction, and macular hole. Ophthalmology. 2013 Dec; 120(12):2611-9.

4 Govetto A, Dacquay Y, Farajzadeh M, Platner E, Hirabayashi K, Hosseini H, et al. Lamellar macular hole: two distinct clinical entities? Am J Ophthalmol. 2016 Apr;164:99-109.

5 Parolini B, Schumann RG, Cereda MG, Haritoglou C, Pertile G. Lamellar macular hole: a clinicopathologic correlation of surgically excised epiretinal membranes. Invest Ophthalmol Vis Sci. 2011;52(12):9074-83.

6 Bottoni F, Deiro AP, Giani A, Orini C, Cigada M, Staurenghi G. The natural history of lamellar macular holes: a spectral domain optical coherence tomography study. Graefes Arch Clin Exp Ophthalmol. 2013 Feb;251(2): $467-75$.

7 Pang CE, Spaide RF, Freund KB. Epiretinal proliferation seen in association with lamellar macular holes: a distinct clinical entity. Retina. 2014;34(8):1513-23.

8 Schumann RG, Compera D, Schaumberger MM, Wolf A, Fazekas C, Mayer WJ, et al. Epiretinal membrane characteristics correlate with photoreceptor layer defects in lamellar macular holes and macular pseudoholes. Retina. 2015 Apr;35(4):727-35.

9 dell'Omo R, Virgili G, Rizzo S, De Turris S, Coclite G, Giorgio D, et al. Role of lamellar hole-associated epiretinal proliferation in lamellar macular holes. Am J Ophthalmol. 2017 Mar;175:16-29.

10 Hubschman JP, Govetto A, Spaide RF, Schumann R, Steel D, Figueroa MS, et al. Optical coherence tomographybased consensus definition for lamellar macular hole. Br J Ophthalmol. 2020 Dec;104(12):1741-7.

11 Pang CE, Spaide RF, Freund KB. Comparing functional and morphologic characteristics of lamellar macular holes with and without lamellar hole-associated epiretinal proliferation. Retina. 2015;35(4):720-6.

12 Compera D, Cereda MG, Schumann RG, Bottoni F. Development and progression of a lamellar macular hole with lamellar hole-associated epiretinal proliferation. Retin Cases Brief Rep. 2019 Fall;13(4):371-5.

13 Tsai CY, Hsieh YT, Yang CM. Epiretinal membrane-induced full thickness macular holes: the clinical features and surgical outcomes. Retina. 2016 Sep;36(9):1679-87.

14 Takahashi H, Kishi S. Tomographic features of a lamellar macular hole formation and a lamellar hole that progressed to a full-thickness macular hole. Am J Ophthalmol. 2000 Nov;130(5):677-9.

15 Tsai CY, Hsieh YT, Yang CM. Epiretinal membrane-induced full thickness macular holes: the clinical features and surgical outcomes. Retina. 2016 Sep;36(9):1679-87.

16 Gass JDM. Idiopathic senile macular hole: its early stages and pathogenesis. Arch Ophthalmol. 1988;106(5): 629-39. 
17 Snead DR, James S, Snead MP. Pathological changes in the vitreoretinal junction 1: epiretinal membrane formation. Eye. 2008;22(10):1310-7.

18 Moshfeghi AA, Flynn HW Jr, Elner SG, Puliafito CA, Gass JD. Persistent outer retinal defect after successful macular hole repair. Am J Ophthalmol. 2005 Jan;139(1):183-4.

19 Powers MA, Shields RA, Moshfeghi AA, Moshfeghi DM. Outer retinal defects represent a normal recovery pathway following internal limiting membrane peeling in macular hole surgery. Ophthalmic Surg Lasers Imaging Retina. 2018 Sep 1;49(9):e1. 